\title{
Investigation of a New Artificial Immune System Model Applied to Pattern Recognition
}

\author{
José Lima Alexandrino, Cleber Zanchettin, Edson C. de B. Carvalho Filho \\ Federal University of Pernambuco \\ Brazil
}

\section{Introduction}

The discovery of new functionalities through the study of human physiology has contributed toward the evolution of Artificial Immune Systems. In this chapter we can investigate a new architecture through observations of natural immunological behaviour, for which application to known algorithms contributed toward an improved performance. It considers a boarding where the antibodies are grouped in an organized way and from an evolutionary process the antibodies that belong to these groupings can improve the adaptive immune reply to a determined antigen. Thus, antibodies of the same class are in the same grouping. Others techniques were implemented such as Clonalg, MLP and K-NN to compare this new model.

\section{Artificial immune systems}

The Artificial Immune Systems (AIS) are a relatively new area of research with considerable potential in helping solve a myriad of difficulties. Its growth has allowed the proposal of new techniques and approaches for solving known problems.

The aim of this technology is to model defence mechanism characteristics and functionalities of living beings. The defence mechanism allows an organism to defend against invasion from foreign substances. The recognition of these substances is based on the key and lock analogy, in which the objective is to find antibodies that have the best immune response to the invading antigens (De Castro \& Timmis, 2002).

The natural immune system stores the best antibodies in its genetic memory. These are later used to identify antigens that have previously invaded the organism, thereby obtaining a quicker, more efficient response.

New functionalities observed in the biological environment were studied for the modelling of this new immunological approach, principally the organization and clustering of similar antibodies $(\mathrm{Ab})$ throughout the process. It is believed that these functionalities may improve the recognition capacity of artificial immune algorithms.

\section{Hybrid architecture}

There are a number of factors that motivate the hybridization of artificial immune algorithms with other techniques. Many complex problems can be decomposed into a

Source: Pattern Recognition Techniques, Technology and Applications, Book edited by: Peng-Yeng Yin, ISBN 978-953-7619-24-4, pp. 626, November 2008, I-Tech, Vienna, Austria 
number of parts, for some of which exact methods, or very good heuristics, may already be available. In these cases it makes sense to use a combination of the most appropriate methods for different sub problems.

In practice frequently, apply those algorithms and evolutionary algorithms (EAs) to a problem where there is a considerable amount of hard-won user experience and knowledge. In such cases performance benefits can often arise from utilizing this information in the form of specialist operators and/or good solutions. Provided that care is taken not to bias the search too much away from the generation of novel solutions. EAs are very good at rapidly identifying good areas of the search space (exploration) and they are less good at the "endgame" of fine-tuning solutions (exploitation) partly as a result of the stochastic nature of the variation operators.

An overall successful and efficient general problem solver, in fact, do not exist. It is commonly experienced that the combination of an evolutionary algorithm and a heuristic method (the hybrid EA) performs better than either of its "parents" algorithms alone (Eiben \& Smith, 2003). For this reason, a Memetic Algorithm (Smith \& Krasnogor, 2005) was developed based on features of natural immune systems. Memetic algorithms are characterized by the hybridization of Evolutionary Algorithms with the use of Local Searches at particular points of the algorithm (Alexandrino \& Carvalho Filho, 2006; Oliveira et al., 2006; Eiben \& Smith, 2003). The initialization method for the main population of antibodies is also a hybridization method, as it employs known solutions (Eiben \& Smith, 2003).

The performance of this model was compared to other techniques found in the literature in order to assess the response quality of the algorithm. MLP (Multi-Layer Perceptron (Rumelhart et al., 1986)), K-NN (K-Nearest Neighbor (Duda \& Hart, 2000)) and Clonalg (Clonal Selection Algorithm (De Castro \& Von Zuben, 2000)) were used. Thus, it was possible to estimate the contribution this new model may have in research regarding artificial immune systems and pattern recognition.

\section{Artificial immune functions}

The discovery of new features from research into the physiology of the human body has contributed toward the evolution of the Artificial Immune System (Dasgupta, 2006). Such architecture has developed the organization and clustering of similar antibodies. The main features of this algorithm are hybridization with local search techniques, the heuristics of population construction, and uses of "intelligent" operators and the generational selection of survivors.

\subsection{Antibody memory}

Smith and Krasnogor (2005) suggest the use of information on previous populations through the retrieval of individuals from past generations. The aim of this technique is to recover genetic information that the algorithm has discarded, functioning as a kind of genetic memory bank to influence the search mechanisms of the algorithm.

This architecture was implemented using genetic memory to investigate the impact of this functionality on the performance of the algorithm.

\subsection{Antibody clustering}

The idea of evolving multiple populations in tandem is also known as island EAs, parallel EAs and more precisely coarse-grain parallel EAs (Eiben \& Smith, 2003). The essential idea 
is to run multiple populations in parallel, in some kind of communication structure. After a (usually fixed) number of generations (known as an epoch), a number of individuals are selected from each population to be exchange with others from neighboring populations this can be thought of as migration.

The original formulation of the GA as a trade-off between exploration of unexplored regions of the search space and exploitation of knowledge gained via search in the vicinity of known high quality solutions. The suggest that during the epochs between communication, when each subpopulation is evolving independently of the others, exploitation occurs, so that the subpopulations each explore the search space around the fitter solutions that they contain. When communication takes place, the injection of individuals of potentially high fitness, and with (possibly) radically different genotypes, facilitates exploration, particularly as recombination happens between the two different solutions.

Whilst extremely attractive in theory, and possessing the highly desirable quality of explicit parallelism, it is obvious that there are no guarantees per se that the different subpopulations are actually exploring different regions of the search space. One possibility is clearly to achieve a start at this through a careful initialization process, but even if this is used, there are a number of parameters that have been shown to affect the ability if this technique to explore different peaks and obtain good results even then when only a single solution is desired as the end result.

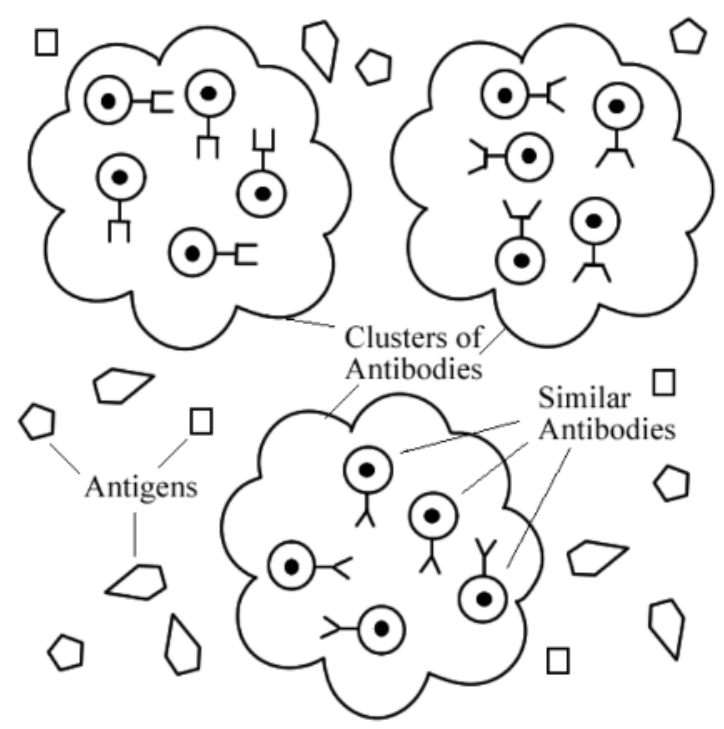

Fig. 1. Clusters with similar antibody

In this specific model, similar antibodies responsible for recognizing a class of antigens are organized into clusters (Fig. 1). With the presentation of an antigen from this class, the antibodies from this cluster multiply and differentiate by means of mutation in order to improve the immune response of the individuals to the antigen class presented. After recognition, the swarm of antibodies undergoes reduction back to its normal size, discarding less apt individuals. The best individuals remain in the swarm of antibodies to improve recognition during future events. 
Genetic memory is organized as islands of evolution (Eiben \& Smith, 2003). This suggests that clusters within sets of similar antibodies are maintained in separate evolution, which allows antibodies to be spatially organized into clusters of individuals in Fig. 1.

Separation allows the antibodies to evolve more quickly, which is different from the approach used in Clonalg (De Castro \& Von Zuben, 2000), where the principal population of antibodies has individuals from a number of different classes. Thus, a more intuitive and better quality evolution is expected in the proposed approach, with antibodies that have a closer affinity to the antigens. This characteristics is important for multi-objective problems. Specifically for pattern recognition like handwritten digit problems.

The individuals of the genetic memory may be newly selected to once again compose the main antibody population, thereby participating in the evolution process. Thus, lost genetic filaments from previous generations can be recovered and reused in future generations.

\section{Immunological model}

This study uses an artificial immune algorithm based on the clonal selection theory and the hill climbing search algorithm (Russell \& Norvig, 2003) as a local search method of the antibodies of the offspring population. The best individuals are stored in a structure based on the adaptive resonance theory.

In the model, similar antibodies responsible for recognizing a class of antigens are organized into clusters. With the presentation of an antigen from this class, the antibodies from this cluster multiply and differentiate by means of mutation in order to improve the immune response of the individuals to the antigen class presented. After recognition, the swarm of antibodies undergoes reduction back to its normal size, discarding less apt individuals. The best individuals remain in the swarm of antibodies to improve recognition during future events.

Genetic memory is organized as islands of evolution (Eiben \& Smith, 2003). This concept suggests that clusters within sets of similar antibodies are maintained in separate evolution, which allows antibodies to be spatially organized into clusters of individuals.

Separation allows the antibodies to evolve more quickly, which is different from the approach used in Clonalg, where the principal population of antibodies has individuals from a number of different classes. Thus, a more intuitive and better quality evolution is expected in the proposed approach, with antibodies that have closer affinity to the antigens. The individuals from the genetic memory may be newly selected to once again compose the main antibody population, thereby participating in the evolution process. Thus, lost genetic filaments from previous generations can be recovered and reused in future generations.

\subsection{Generation of the populations}

In the present approach, it was determined that the main antibody population would be composed of $\lambda=20$ individuals and the offspring population would have 40 individuals. The memory population is the same size as the training set and is generated from a selection of the best individuals.

At the beginning of the algorithm, the main population is generated randomly. With the next generation, the main population is composed of $50 \%$ random individuals and $50 \%$ individuals from the genetic memory of the antigen class that is being presented. This type of formation helps maintain the diversity of the main antibody population and allows the reuse of lost genetic material to initialize the main population with individuals of high affinity (Eiben \& Smith, 2003). 
Each individual of the offspring population is generated from a size-2 tournament selection (Eiben \& Smith, 2003) of the antibodies in the main population. The antibody chosen undergoes the application of the mutation operators.

This type of main population generation seeks to model a biological generational characteristic in the human organism. When an antigen is presented, the multiplication of antibodies in that set occurs in order to find the antibody that has the best response to the antigen. This type of initialization of the main population allows the algorithm to retrieve individuals from the genetic memory bank that may have been discarded during a survival selection based on fitness (Eiben \& Smith, 2003).

\subsection{Memory population}

De Castro \& Von Zuben (2000) propose Clonalg using a selection of survivors proportional to fitness, in which only the most apt antibodies survive to the next generation. In the present study, the antibody population is generational (Eiben \& Smith, 2003). This choice was made to avoid the premature convergence of the algorithm in regions of local minimums due to the highly elitist choice of antibodies used in Clonalg.

This point of the algorithm is determined by the add antibody to population memory Fig. 3 and constitutes the memory population, which is responsible for the learning of the training antigens.

At each iteration, the best antibody in the principal and offspring populations is selected by the flow chart demonstrated in Fig. 3. This antibody is the candidate to integrate the memory population. If it possesses better fitness than an individual in the memory population, it will replace it. The added antibody is responsible for recognizing the antigen presented by the training population. There will be one antibody in the memory population associated to each antigen of the training population.

Following this step, the individual is added to the genetic memory of the class of training antigen that is being presented. There is a genetic memory for each class of the training set. This memory contains all the antibody candidates from the memory population that were selected during the execution of the algorithm. The set of antibodies will be used to compose the main population of upcoming generations.

\subsection{Populevaluation}

The quality of each antibody in the population is measured by the Hamming Distance DH (De Castro \& Timmis, 2002) calculation between it and the antigen that is being presented, according to (1). Shorter distances equal greater quality.

Considering $\mathrm{N}$ the set of antigens $(\mathrm{Ag}) \mathrm{Ag}_{\mathrm{i}}, \mathrm{i}=1, \ldots, \mathrm{L}\left(\mathrm{Ag}_{\mathrm{i}} \in \mathrm{N}\right)$ to be recognized, and $\mathrm{P}$ the set of antibodies $(\mathrm{Ab}) \mathrm{Ab} b_{i}, \mathrm{i}=1, \ldots, \mathrm{L}\left(\mathrm{Ab} b_{\mathrm{i}} \in \mathrm{P}\right)$ to be used as pattern recognizers. The antigens and antibodies have the same length $\mathrm{L}$.

$$
D_{H}=\sum_{i=1}^{L} \delta, \text { where } \delta=\left\{\begin{array}{l}
1 \text { if } A_{i} \neq A b_{i} \\
0 \text { otherwise }
\end{array}\right.
$$

\subsection{Mutation operators}

Two mutation operators were employed in the present study: simpleMutation and totalMutation. With the first operator, one gene is chosen from all possible genes and only this gene will undergo mutation (Eiben \& Smith, 2003). 
With the second operator, all genes have a 30\% likelihood of undergoing mutation (Eiben \& Smith, 2003).

All antibodies have $30 \%$ likelihood of mutation application. This variable determines if an individual will undergo mutation or return to its copy.

\subsection{Local search}

The Hill Climbing (HC) Search Algorithm (Russell \& Norvig, 2003) is a local search and is shown in Fig. 2. It is simply a loop that continually moves in direction of increasing quality value.

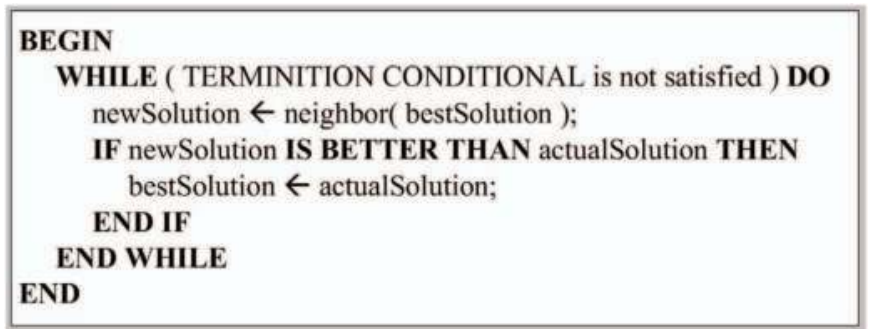

Fig. 2. The general schema of a search in pseudocode

This architecture opts to implement the local search after the mutation operations and before the selection of survivors, as described in Fig. 3.

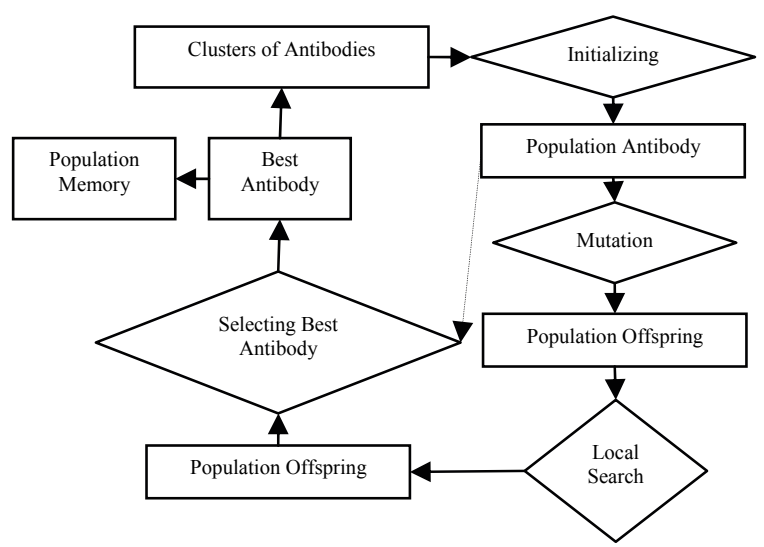

Fig. 3. Flow chart of the algorithm

\subsection{Description of the algorithm}

With each presentation of an antigen, a new main population is created. From this, a new offspring population is formed through the application of mutation operators. The best antibody found by the two populations is the candidate for incorporating the memory population of antibodies. This antibody will automatically be added to the genetic memory of the antigen class that is being added. At the end of the presentation of all the training patterns, a generation of the algorithm will be considered. 
At the end of each generation, the classification rate in the test set is calculated in relation to the memory population of antibodies. The best configuration of the memory population is stored until the stopping criterion of the algorithm is satisfied. Only the maximum number of generations was considered in this implementation.

At the end of each generation, the classification rate in the test set is calculated in relation to the memory population of antibodies. The best configuration of the memory population is stored until the stopping criterion of the algorithm is satisfied. Only the maximum number of generations was considered in this implementation.

\section{Recognition of handwritten digits}

The analyzed patterns consist of binary images of handwritten digits from 0 to 9 . The database used in this analyse corresponds to 8000 handwritten digit patterns divided into two sets of 6000 training patterns and 2000 test patterns. Each one of the ten classes had the same number of patterns.

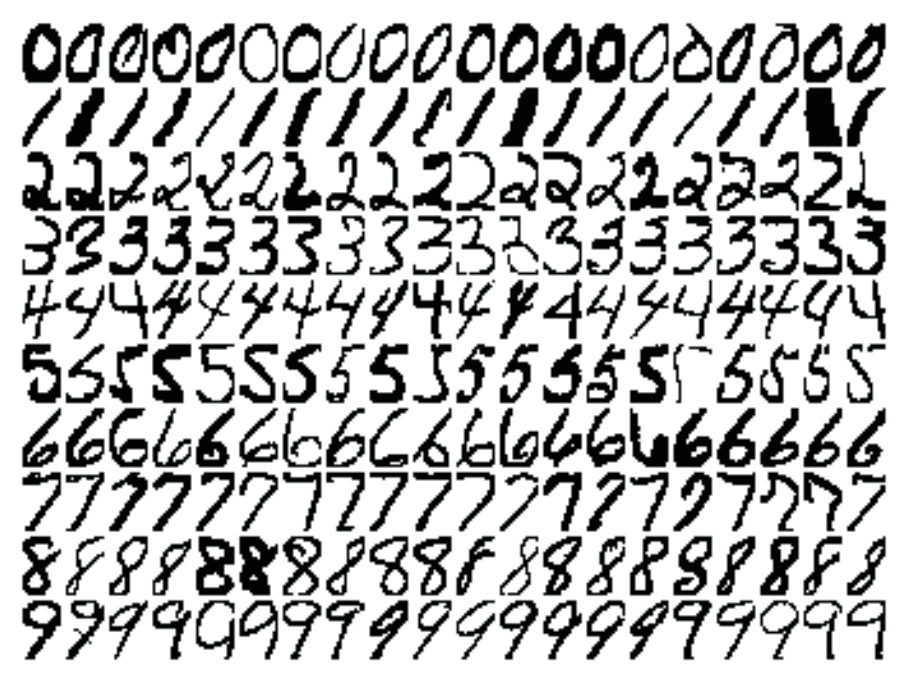

Fig. 4. Examples of patterns used

The patterns have $24 \times 16$ pixels and were transformed into binary matrices with 24 lines and 16 columns. An individual is composed of 24 genes, corresponding to a Size- 24 vector that is the number of input features. Each element of the vector corresponds to each line of the binary matrices and one gene in the pattern. Each gene was formed by a number with binary form and size 16 that corresponds to a line of the binary matrix. This number was transformed into base 10 .

\section{Experiments and results}

In the neural network training the aim is to achieve topologies with few connections and small error. In these tests, an MLP was used with only one intermediate layer and a Sigmoid Logistic Function. The MLP contains all possible connections of forward propagation 
(feedforward) between adjacent layers, without having connections that link the processing units of non-adjacent layers. In network training, considering $\mathrm{N}_{\mathrm{C}}$ classes in the data set, the true class of the pattern $\mathrm{x}$ from the training set $\mathrm{Pt}$ is defined as:

$$
\gamma(x) \in\left\{1,2, \ldots, N_{C}\right\}, \forall x \in P_{t}
$$

In the experiment, the winner-takes-all classification rule was used, in which the number of output units (N3) is equal to the number of classes $\left(\mathrm{N}_{\mathrm{C}}\right)$.

As $o_{k}(x)$ is the output value of the output unit $k$ for the pattern $x$, the class assigned to pattern $x$ is defined as:

$$
\varphi(\mathrm{x})=\operatorname{argmaxo}_{\mathrm{k}}(\mathrm{x}), \forall \mathrm{x} \in \mathrm{P}_{\mathrm{t}}, \mathrm{k} \in\left\{1,2, \ldots, \mathrm{N}_{3}\right\}
$$

The network error for the pattern $\mathrm{x}$ is defined as follows:

$$
\varepsilon(x)=\left\{\begin{array}{l}
1, \text { if } \varphi(x) \neq \gamma(x) . \\
0, \text { if } \varphi(x)=\gamma(x) .
\end{array}\right.
$$

Therefore, the classification error for the training set $\mathrm{Pt}$, which represents the percentage of incorrectly classified training patterns, can be defined as:

$$
E\left(P_{t}\right)=\frac{100}{\# P_{t}} \sum_{x \in P_{t}} \varepsilon(x)
$$

where \#Pt is the number of patterns in the set Pt.

The percentage of connections used by the network is given by:

$$
\Psi(C)=\frac{100}{N_{\max }} \sum_{i=1}^{N_{\max }} c_{i}
$$

The training process stops if: (1) the GL5 criterion defined in Proben1 (Prehelt, 1994) is met (based on the classification error or SEP of the validation set); or (2) the maximum number of iterations is reached. For the implementation of the GL5 criterion, the classification error or SEP for the validation set is evaluated at each IT iterations.

The GL5 criterion is a good approach for avoiding overfitting to the training set. The classification error for the validation set $\mathrm{Pv}$ is given by $\mathrm{E}(\mathrm{Pv})$, which is calculated according to Equation (5). Thus, using $\mathrm{V}(\mathrm{k})$ to denote the classification error $\mathrm{E}(\mathrm{Pv})$ at iteration $\mathrm{i}=\mathrm{kI}_{\mathrm{T}}$, $\mathrm{k}=1,2, \ldots, \mathrm{I}_{\max } / \mathrm{I}_{\mathrm{T}}$, the generalization loss parameter $(\mathrm{GL})$ is defined as the relative increase in the validation error over the minimum-so-far. The GL5 criterion stops the execution when the parameter GL becomes higher than $10 \%$.

$$
G L(k)=\left(\frac{V(k)}{\min _{j<k} V_{j}}-1\right)
$$


To choose a data set of training patterns for the K-NN was used uniform distribution with maximum size of training patterns (600). For each execution a new set of training is chosen. Liu et al., 2003 state that the quality of the K-NN classifier is directly influenced by the $\mathrm{k}$ number of its nearest neighbors. Therefore, all training patterns are used as prototypes in the present analysis.

\begin{tabular}{|ccccc|}
\hline$\#$ & Architecture & Clonalg & K-NN & MLP \\
\hline 1 & {$[46.43,47.57]$} & {$[38.44,40.43]$} & {$[41,97,43,03]$} & {$[87.36,93.84]$} \\
2 & {$[48.74,49.73]$} & {$[39.00,40.74]$} & {$[47,45,48,22]$} & {$[88.24,93.89]$} \\
4 & {$[56.46,57.41]$} & {$[42.82,44.31]$} & {$[56,34,57,40]$} & {$[88.77,94.43]$} \\
8 & {$[60.72,61.81]$} & {$[44.73,45.87]$} & {$[58,89,60,04]$} & {$[90.94,97.13]$} \\
16 & {$[63.81,64.73]$} & {$[47.92,48.54]$} & {$[66,19,67,47]$} & {$[90.63,96.64]$} \\
32 & {$[72.83,73.50]$} & {$[52.65,53.35]$} & {$[74,93,75,74]$} & {$[91.68,97.38]$} \\
64 & {$[76.02,77.11]$} & {$[53.62,55.05]$} & {$[79,84,80,90]$} & {$[90.02,95.91]$} \\
\hline
\end{tabular}

Table 1. Analysis of algorithm performance in relation to aggregate training performance Each algorithm was executed with the same training data, but of different sizes. This served to test the generalization capacity of each algorithm under situations of different quantities of training patterns and analyze their performance. Training sets of the following sizes were used: 1, 2, 4, 8, 16, 32 and 64 patterns per class. For all cases, the classes always have the same number of patterns. The test set has the same 100 patterns for each class. At the end of the training step, the classification rate is calculated for the four algorithms analyzed.

Each algorithm was executed 30 times to obtain the classification rate. Table 1 displays the confidence intervals of the samples for the four algorithms studied. These intervals serve for a grounded comparison between algorithms. The confidence level considered was $95 \%$.

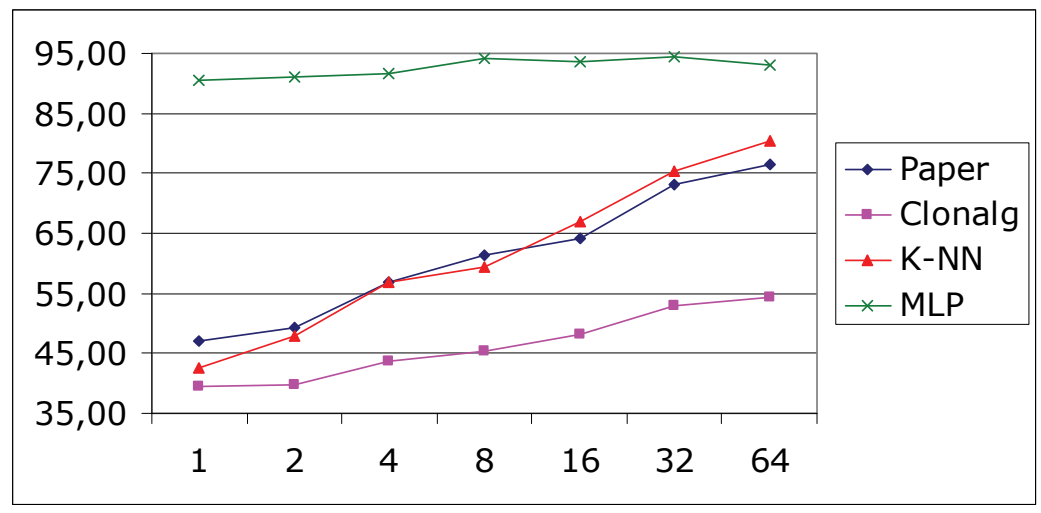

Fig. 5. Evolution of classification rate 
The present study demonstrates that the new approach is both more biologically plausible and obtains better results than other AIS, such as clonalg.

Algorithm performance is measured through the classification rate in the data set of test patterns.

As Fig. 5 shows, the proposed algorithm obtained similar results to K-NN. This performance proximity between the proposed architecture and K-NN, with a comparison to Clonalg, suggests that the new approach makes a significant contribution toward improving the results. Considering the average value of the recognition rate, the algorithm proposed obtained a good performance. Analyzing the computational cost of the four algorithms, the MLP presented the best cost-benefit in providing training time when compared to the other algorithms. Despite having the best recognition rate, the architecture proposed had the longest training time of all the algorithms.

Depending on the type of problem studied, the time available for training can be a critical variable. Regarding the analysis of test patterns, this approach, Clonalg and MLP presented similar times. K-NN obtained the longest time of all due to its high computational cost.

\section{Conclusions}

This chapter describes an application based on Artificial Immune System (AIS) with biologically inspired characteristics, such as the grouping of similar antibodies and memory antibodies were studied to allow the evolution of the AIS. The focus of this chapter was to evaluate the quality of this model to recognize handwritten digits patterns and evolving its performance and comparing it to other technique.

This architecture combined different techniques to form a hybrid immune algorithm. It was biologically inspired, with the use of the Clonal selection principle as a method for propagating the genetic material of the individuals. Biological memory proved to be an efficient functionality for hybridization in the generation of the main population, thereby constituting a more biologically plausible evolutionary view.

The selection of generational survivors proved more biologically plausible, suggesting the organization of antibodies into clusters during the evolution process. This model allowed a more intuitive evolution of antibodies. All the antibodies created during the evolutionary process are stored in an antibody memory in order to avoid the loss of good individuals. It is possible to use good genetic material in the future by employing antibodies from past generations. The best antibodies are responsible for the recognition of the set of test antigens and these individuals will be organized into clusters.

The selection of generational survivors proved more biologically plausible, suggesting the organization of antibodies into clusters during the evolution process. This model allowed a more intuitive evolution of antibodies.

One advantage of this model is the possibility of adaptation to new patterns through the preservation of previously acquired knowledge while continuing to learn new knowledge. This study offers a good contribution to the literature. It presents an efficient model in comparison to other artificial immune systems, thereby contributing significantly toward an improvement in the results. 


\section{Acknowledgment}

The authors would like to thank CNPq and CAPES (Brazilian research agencies) for their financial support.

\section{References}

Alexandrino, J. L. \& Carvalho Filho, E. C. B. (2006). Investigation of a new artificial immune system model applied to pattern recognition. Proceedings of International Conference on Hybrid Intelligent Systems, Auckland, New Zealand, December, 2006, pp. 16-20.

Alexandrino J. L. ; Zanchettin C. \& Carvalho Filho, E. C. B. (2007) Artificial Immune System with ART Memory Hibridization. Proceedings of International Conference on Hybrid Intelligent Systems, Kaiserslautern, Germany, September, 2007, pp. 59-64.

Carpenter, G. A. \& Grossberg, S. (1986). A massively parallel architecture for a selforganizing neural pattern recognition machine. Computer Vision, Graphics, and Image Processing, Vol 37, pp. 54-115.

Dasgupta, D. (2006). Advances in Artificial Immune Systems. IEEE Computational Intelligence Magazine.

De Castro, L. N. \& Von Zuben, F. J. (2000). The Clonal Selection Algorithm with Engineering Applications, Proceedings of Genetic and Evolutionary Computation Conference, Las Vegas, Nevada, USA, July, 2000, pp. 36-37.

De Castro, L. N. \& Timmis, J. I. (2002). Artificial Immune Systems: A Novel Paradigm for Pattern Recognition, In : Artificial Neural Networks in Pattern Recognition, L. Alonso, J. Corchado, C. Fyfe, 67-84, University of Paisley.

Duda, R. O. \& Hart, P. E. (2000). Pattern Classification, John Wiley and Sons, New York.

Eiben, E. \& Smith, J. E. (2003). Introduction to Evolutionary Computing, In: Natural Computing Series, MIT Press, Springer, Berlin.

Liu, C. L. ; Nakashima, K. ; Sako, H. \& Fujisawa, H. (2003) Handwritten Digit Recognition: Benchmarking of State-of-the-Art Techniques, Pattern Recognition, Vol. 36, No. 10, October 2003, 2271-2285.

Moscato, P. (1989) On evolution, search, optimization, GAs and martial arts: toward memetic algorithms, California Institute Technology., Pasadena, CA, Tech. Rep. Caltech Concurrent Comput. Prog. Rep. 826, (1989).

Oliveira, H. C. B. ; Alexandrino, J. L. \& Souza M. M. Memetic and genetic algorithms: A comparison among different approaches to solve vehicle routing problem with time windows. Proceedings of International Conference on Hybrid Intelligent Systems, Auckland, New Zealand, December, 2006, pp. 55-60.

Ong, Y.-S.; Lim, M.-H.; Zhu, N. and Wong, K.-W. (2006) Classification of Adaptive Memetic Algorithms: A Comparative Study, IEEE Transactions on Systems, Man, and Cybernetics - part B: Cybernetics, Vol. 36, No. 1, February 2006.

Prehelt, L. (1994). Proben1 - a set of neural network benchmark problems and benchmarking rules. Technical Report 21, University of Karlsruhe. 
Rumelhart, D. E.; Hinton, G. E. \& Williams, R. J. (1986). Learning internal representations by error propagation, In : Parallel Distributed Processing, Vol. 1, pp. 318-362, Cambridge, MIT Press.

Russell, S. J. \& Norvig, P. (2003) Artificial Intelligence: A Modern Approach. Prentice Hall.

Smith, J. \& Krasnogor, N. (2005). A Tutorial for Competent Memetic Algorithms: Model, Taxonomy, and Design Issues. IEEE Transactions on Evolutionary Computation, Vol. 9, No. 5, October 2005, 474-488. 


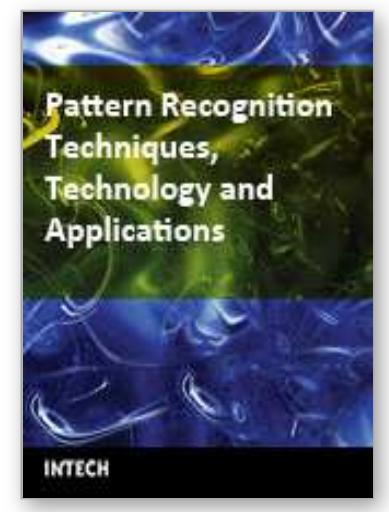

\author{
Pattern Recognition Techniques, Technology and Applications \\ Edited by Peng-Yeng Yin
}

ISBN 978-953-7619-24-4

Hard cover, 626 pages

Publisher InTech

Published online 01, November, 2008

Published in print edition November, 2008

A wealth of advanced pattern recognition algorithms are emerging from the interdiscipline between technologies of effective visual features and the human-brain cognition process. Effective visual features are made possible through the rapid developments in appropriate sensor equipments, novel filter designs, and viable information processing architectures. While the understanding of human-brain cognition process broadens the way in which the computer can perform pattern recognition tasks. The present book is intended to collect representative researches around the globe focusing on low-level vision, filter design, features and image descriptors, data mining and analysis, and biologically inspired algorithms. The 27 chapters coved in this book disclose recent advances and new ideas in promoting the techniques, technology and applications of pattern recognition.

\title{
How to reference
}

In order to correctly reference this scholarly work, feel free to copy and paste the following:

José Lima Alexandrino, Cleber Zanchettin and Edson C. de B. Carvalho Filho (2008). Investigation of a New Artificial Immune System Model Applied to Pattern Recognition, Pattern Recognition Techniques, Technology and Applications, Peng-Yeng Yin (Ed.), ISBN: 978-953-7619-24-4, InTech, Available from: http://www.intechopen.com/books/pattern_recognition_techniques_technology_and_applications/investigation_ of_a_new_artificial_immune_system_model_applied_to_pattern_recognition

\section{INTECH}

open science | open minds

\section{InTech Europe}

University Campus STeP Ri

Slavka Krautzeka 83/A

51000 Rijeka, Croatia

Phone: +385 (51) 770447

Fax: +385 (51) 686166

www.intechopen.com

\section{InTech China}

Unit 405, Office Block, Hotel Equatorial Shanghai

No.65, Yan An Road (West), Shanghai, 200040, China

中国上海市延安西路65号上海国际贵都大饭店办公楼 405 单元

Phone: +86-21-62489820

Fax: $+86-21-62489821$ 
(C) 2008 The Author(s). Licensee IntechOpen. This chapter is distributed under the terms of the Creative Commons Attribution-NonCommercialShareAlike-3.0 License, which permits use, distribution and reproduction for non-commercial purposes, provided the original is properly cited and derivative works building on this content are distributed under the same license. 